\title{
Myelomeningocele Knee Flexion Contracture: Posterior Capsulectomy and Hamstring Lengthening
}

\author{
Bruno Casaccio, Silvia Reciniello and Mario Lampropulos* \\ Traumatology and Orthopedics, Hospital Italiano de Buenos Aires, Argentina \\ *Corresponding author: Mario Lampropulos, Traumatology and Orthopedics, Hospital Italiano de Buenos Aires, Argentina. \\ To Cite This Article: Mario Lampropulos. Myelomeningocele Knee Flexion Contracture: Posterior Capsulectomy and Hamstring Lengthening. \\ Am J Biomed Sci \& Res. 2019 - 2(4). AJBSR.MS.ID.000595. DOI: 10.34297/AJBSR.2019.02.000595
}

Received: April 03, 2019 | Published: April 18, 2019

\section{Abstract}

Introduction: Knee flexion contracture is a common problem in Myelomeningocele patients. Disturb and limits walking abilities; promote Crouch Gait and increase energy cost, interfering with child's walking efficiency. Several treatments have been proposed, the aim of our study is to evaluate the results obtained with posterior capsulectomy, hamstring lengthening and patellar tendon advancement.

Materials and methods: A retrospective study was carried out in which we included all Myelomeningocele patients who underwent surgery for knee flexion contracture with posterior capsulectomy, hamstring lengthening and patellar advancement; between 2008 and 2016, with a minimum follow up of 1 year. Preoperative and postoperative goniometric measures were taken according to the methods described by Norkin and White. Clinical controls and regular follow-up were carried out throughout the follow-up.

Results: 15 Patients (26 knee). 11 male patients (73.3\%) and 4 females (26.6\%), with an average age of 11.93 years. 11 of the patients were ambulators and 4 patients were non-ambulators. Mean flexion angle measured preoperatively was $41,07^{\circ}$, it decreases after surgery to a mean of $5,42^{\circ}$. All the patients had a postoperative flexion angle less than $15^{\circ}$. A statistically significant change was observed in the pre and postoperative angles.

Conclusions: we believed that posterior capsulectomy, hamstring lengthening, and patellar tendon advancement is a good alternative for the treatment of knee flexion contracture in Myelomeningocele patients. We recommend the surgical release of flexion contracture, when this is greater than $15^{\circ}$ and generates impediments to walking, standing, sitting positions or transfers.

\section{Introduction}

Knee flexion contracture is a common problem in Myelomeningocele patients, whit higher incidence in patients with lower thoracic and high lumbar lessons [1,2]. It is produced by several factors, among which, the static forces of positioning and the muscular imbalance are the most important [3]. Disturb and limits walking abilities [4], promote Crouch Gait, and increases energy cost, interfering with child walking efficiency. For these reasons, surgical release has been recommended when a knee flexion contracture exceeds $15^{\circ}$ [5].

Several treatments have been proposed for this deformity, as tendon lengthening, capsular releases, or supracondylar femoral extension osteotomies [6] and also distal anterior femoral hemipifisiodesis for guided growth correction [7]. The aim of our study is to evaluate the results obtained with posterior capsulectomy, hamstring lengthening and patellar tendon advancement.

\section{Material and Methods}

A retrospective study was carried out in which we included all myelomeningocele patients treated in our institution for correction of knee flexion contracture with posterior capsulectomy, hamstring lengthening, and patellar tendon advance between 2008 and 2016, with a minimum follow up of 1 year.

Patients that were treated with other techniques have been excluded of the study (supracondylar femoral extension osteotomies, hamstring lengthening without capsulectomy). All interventions had been carried out by the same Neuro-orthopedic surgeon (ML). Clinical goniometric measurements were made preoperative and then in the postoperative period after removal of the immobilization at 4 weeks. All measurements were made according to the methods described by Norkin \& White [8], the patient in supine position, the knee in maximum extension and the hip neutral. Radiographs were made in the immediate postoperative period to all patients to evaluate implanted surgical material. Clinical regular controls were carried out throughout the follow-up.

\section{Surgical Technique}

The patients in dorsal decubitus, posterolateral knee incision is made, the external popliteal sciatic nerve is repaired and we performed a selective fascial lengthening of the femoral biceps 
(Figure 1), the release of the external gastrocnemius head, and subsequent capsulectomy, which consists of the resection of a square of $1 \mathrm{~cm}$ per side at the level of the posterior femoral-tibial joint. A posteromedial knee incision was then made, through which a selective facial lengthening of Semitendinosus and Semimembranosus was carried out, the release of the internal gastrocnemius head and the posterior capsulectomy as described on the external side (Figure 2). The patient was then torn to the lateral decubitus position and with the knee in extension, an anterior incision was made from the distal pole of the patella to the anterior tuberosity of the tibia., we identified there the patellar tendon, that is seen loose, advancement was performed with a harpoon placed below the tuberosity of the tibia, and was controlled with intraoperative x-ray.

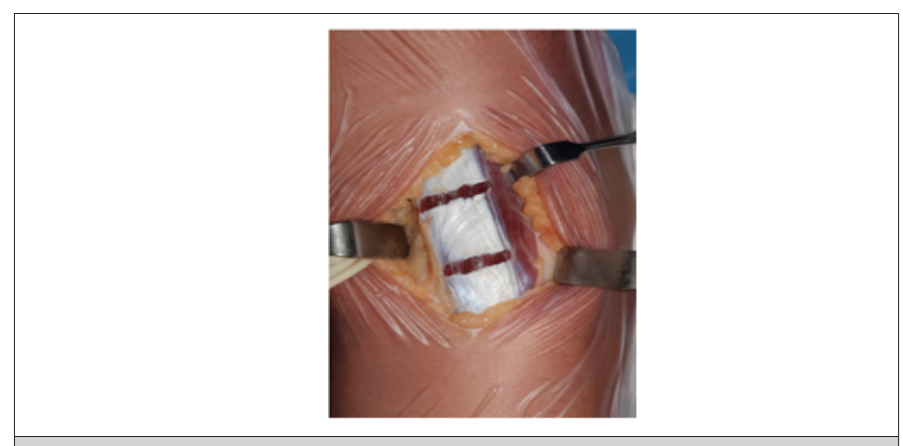

Figure 1.

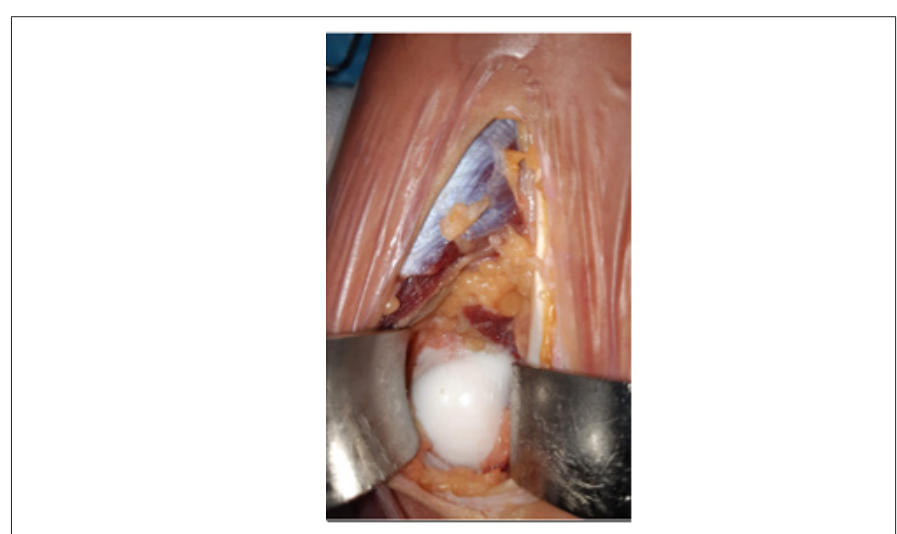

Figure 2.

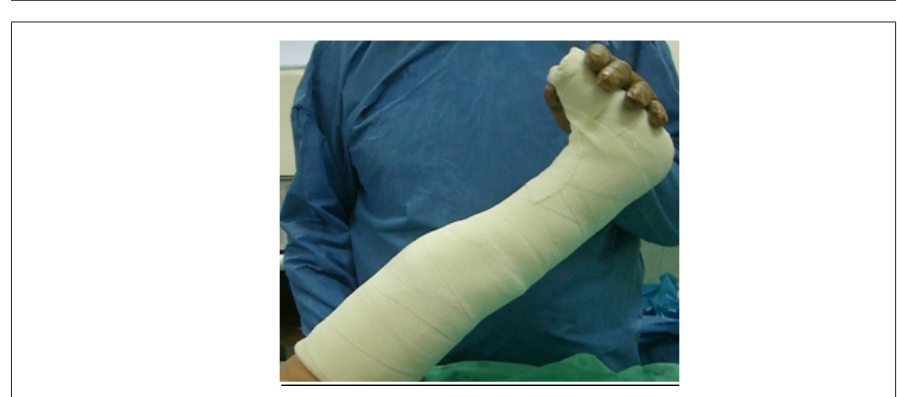

Figure 3.

Inguinopedic plaster immobilization was performed in maximum knee extension, with ankle at $90^{\circ}$ and neutral rotation and maintained over 4 weeks (Figure 3), windows plaster cures were conducted during this period. In some cases where surgery was part of multilevel procedures the plaster was also adapted to the procedures performed on hips and knees.
After the fourth postoperative week, the cast is removed, patients began with rehabilitation, and orthoses were indicated according to the need of the patients regarding their muscle values during day, and long night KAFO ortesis to avoid knee flexion at rest (Figure $4 \& 5$ ). Weight load bipedestation and walking was allowed after plaster removal and began with gait rehabilitation in kinesiology.

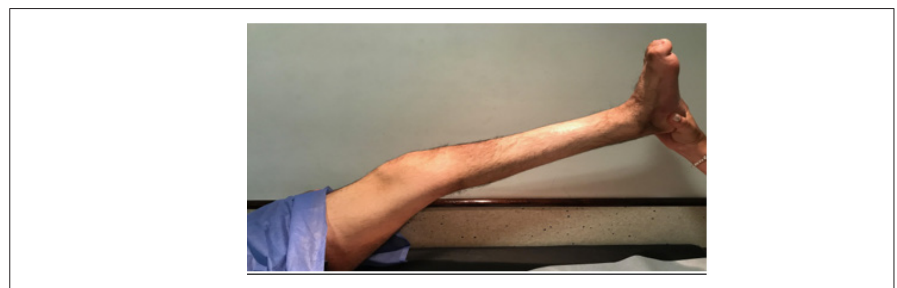

Figure 4. Preoperative flexion contracture.

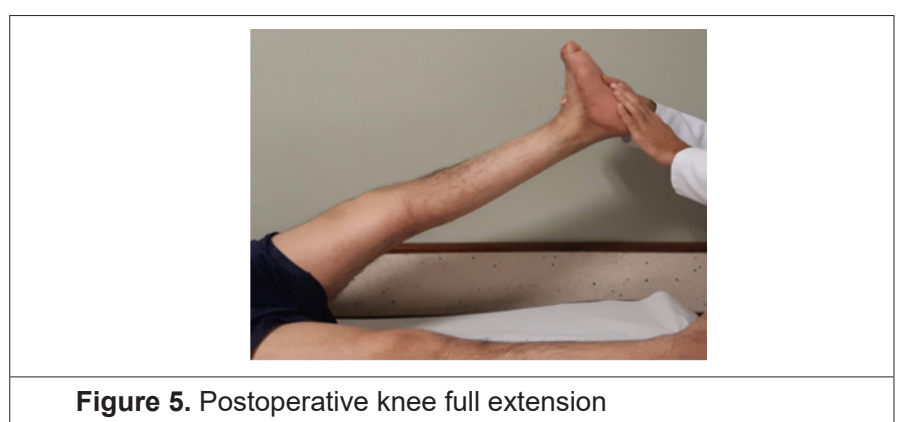

\section{Results}

The sample had 15 patients, totalling 26 knees intervened, 11 male patients $(73.3 \%)$ and 4 women (26.6\%), with an average age of 11.93 years, with a range of 5 to 28 years. The average follows up was 53.26 months, ranging between 12 and 92 months.

11 of the patients were ambulatory and 4 patients were nonambulatory, regarding the GMFCS, we have 6 class III patients and 9 class IV patients. Only one patient improved in his GMFCS, being IV in the preoperative and III in the postoperative period. 6 of the patients had previous treatments, 3 with soft tissue surgeries without capsulectomy and 3 with supracondylar femoral extension osteotomies. 8 patients in our sample were operated on his knees in context of multilevel surgery.

We performed a statistical analysis of the results obtained with a Wilcoxon signed rank test which is a non-parametric test to compare the average range of two related samples (or not) and determine if there are differences between them. It is used as an

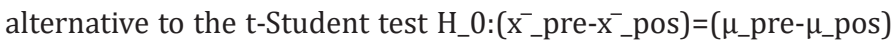
when the normality of these samples cannot be assumed. This test also allows to evaluate if a sample comes from a theoretical population, replacing the t-Student test to evaluate if the sample media $\bar{x}$ is equal to the population media $\mu$. The tests and graphics were done with the software R i386 3.4.0.

The mean of the flexion angle measured preoperatively was $41.07^{\circ}$, which after surgery decreased to an average of $5.42^{\circ}$ (Graphic 1). All the operated patients had a postoperative flexion angle less than $15^{\circ}$. A statistically significant change was observed in terms of pre and post-operative angles $(p<0,0001)$ (Table 1$)$, 
noting that the postoperative angles on average approach the normal values of correction $\left(0^{\circ}-10^{\circ}\right)$.

\begin{tabular}{|l|l|l|}
\hline & \\
\hline & \\
\hline
\end{tabular}

\begin{tabular}{|c|c|c|}
\hline \multicolumn{2}{|l|}{ Table 2. } \\
\hline Patients & Media \pm SD $\left({ }^{\circ}\right)$ & p-value 3 \\
\hline Recurrence $(n=6)$ & $49,66 \pm 12,596$ & \multirow{2}{*}{0,0827} \\
\hline No recurrence $(n=20)$ & $38,50 \pm 11,596$ & \\
\hline
\end{tabular}

We found in the follow up, 3 patients who presented recurrences, 2 of which were re-operated surgically to achieve the correction, the remaining patient did not want to perform the new surgery indicated, so an expectant behaviour was taken.

We made a comparison of preoperative and postoperative flexion angles in relation to the recurrences observed and found that the average preoperative flexion angle in patients who later had recurrences was $49.66^{\circ}$, while the average of the operated patients who did not have recurrences was $38.5^{\circ}$ (Table 2 ). This difference is not statistically significant, with a $\mathrm{p}=0,08$, but it is noteworthy that the average of the previous angles was higher in those patients who had recurrences and when obtaining a p-value close to the critical value $\alpha$, considering the small size of the sample, this result should not be taken as definitive.

Considering postoperative angles in patients with recurrence, these were on average of $7^{\circ}$, in those patients who did not present recurrence were on average of $4.95^{\circ}$. No statistically significant difference was observed between these angles $(p=0,25)$.

We found in the postoperative period 2 complications, both of them presented ulcers by decubitus in the talar region due to immobilization with plaster. They required treatments with hydrocolloids. Neither patients required toilletes or antibiotics. The healing of the wound was complete, with no subsequent sequelae.

\section{Discussion}

Knee flexion contracture is a well-known and documented deformity in patients with myelomeningocele. Produces Crouch Gait and limitations in mobility, increasing walking energy cost and decreasing the quality of life of patients.

In our series of patients, we evaluate the improvement in the degree of extension of postoperative knee, these results are comparable with those obtained by HK Graham [1] who presents
28 patients that underwent hamstring lengthening and added capsulectomy in those who required greater release, with an average of flexion contracture in preoperative of $39^{\circ}$ and postoperative of $5^{\circ}$ immediate and $13^{\circ}$ in the remote follow-up, demonstrating also the improvement in gait and transfers achieved by surgical release of the contracture.

Dias et al. [5] in their study, performed capsulectomies and hamstring lengthening in 11 patients, achieving an improvement in flexion contracture from $25^{\circ}$ preoperatively to $6^{\circ}$ postoperatively, measures by gait analysis also showed dynamic sagittal kinematics improvements during the initial contact and midstance phase.

There are limitations in our study. First, those inherent to its retrospective nature. Also, the measurements were made clinically, and it has been shown that there are certain differences between this form of measurement and the actual contracture during walking. [9], which would suggest that measurement by gait analysis could be more reliable.

\section{Conclusions}

We believe that posterior Capsulectomy, lengthening of the hamstrings and patellar tendon advance technique is an alternative for the treatment of knee flexion contracture in patients with myelomeningocele. We obtained positive postoperative results in our series, with statistically significant changes and achieving correction values that reduce the limitations of walking and transfers, with a low rate of complications and recurrences.

We recommend the surgical release of knee flexion contracture, when this is greater than $15^{\circ}$ and generates impediments on walking, standing, sitting positions or transfers.

\section{References}

1. Marshall PD, Broughton NS, Menelaus MB, Graham HK (1996) Surgical release of knee flexion contractures in Myelomeningocele. J Bone Joint Surg 78(6): 912-916.

2. Moen T, Gryfakis N, Dias L, Lemke L (2005) Crouched Gait in Myelomeningocele: A Comparison Between the Degree of Knee Flexion Contracture in the Clinical Examination and During Gait. J Pediatr Orthop 25(5): 657-660.

3. Vineeta T Swaroop and Luciano Dias (2009) Orthopedic management of spina bifida. Part I: hip, knee, and rotational deformities. J Child Orthop 3: 441-449.

4. Dias, Luciano S (1992) Surgical Management of Knee Contractures in Myelomeningocele. Journal of Pediatric Orthopedics 2(2): 127-131.

5. Moen TC, Dias L, Swaroop VT, Gryfakis N, Kelp-Lenane C (2011) Radical Posterior Capsulectomy Improves Sagittal Knee Motion in Crouch Gait. Clin Orthop Relat Res 469(5): 1286-1290.

6. Pablo Bergamo, Miguel Puigdevally, Mario Lamprópulos (2005) Mielomeningocele. Rev Asoc Argent Ortop Traumatol 70: 269-283.

7. Al-Aubaidi Z, Lundgaard B, Pedersen NW (2012) Anterior distal femoral hemiepiphysiodesis in the treatment of fixed knee flexion contracture in neuromuscular patients. J Child Orthop 6(4): 313-318.

8. Cynthia C Norkin, D Joyce White (2004) Measurement of Joint Motion: A guide to Goniometry. Jaypee Brothers Medical Publishers, India.

9. Moen T, Gryfakis N, Dias L, Lemke L (2005) Crouched Gait in Myelomeningocele: A Comparison Between the Degree of Knee Flexion Contracture in the Clinical Examination and During Gait. Pediatr Orthop 25(5): 657-660. 